\title{
VISIBLE ACTIONS ON FLAG VARIETIES OF TYPE C AND A GENERALIZATION OF THE CARTAN DECOMPOSITION
}

\author{
YUICHIRO TANAKA
}

(Received March 12, 2012, revised June 18, 2012)

\begin{abstract}
We give a generalization of the Cartan decomposition for connected compact Lie groups of type $\mathrm{C}$ motivated by the work on visible actions of T. Kobayashi [J. Math. Soc. Japan, 2007] for type A groups. Let $G$ be a compact simple Lie group of type C, $K$ a Chevalley-Weyl involution-fixed point subgroup and $L, H$ Levi subgroups. We firstly show that $G=L K H$ holds if and only if either Case I: $(G, H)$ and $(G, L)$ are both symmetric pairs or Case II: $L$ is a Levi subgroup of maximal dimension and $H$ is an arbitrary maximal Levi subgroup up to switch of $L, H$. This classification gives a visible action of $L$ on the generalized flag variety $G / H$, as well as that of the $H$-action on $G / L$ and of the $G$-action on the direct product of $G / L$ and $G / H$. Secondly, we find a generalized Cartan decomposition $G=L B H$ explicitly, where $B$ is a subset of $K$. An application to multiplicity-free theorems of representations is also discussed.
\end{abstract}

1. Introduction and statement of main results. In this paper, we classify all the pairs of Levi subgroups $(L, H)$ of a connected compact simple Lie group $G$ of type $\mathrm{C}$ such that $G=L G^{\sigma} H$ holds, where $\sigma$ is a Chevalley-Weyl involution of $G$. The motivation for considering this kind of decomposition is the theory of visible actions on complex manifolds introduced by Kobayashi [6], and $G=L G^{\sigma} H$ can be interpreted as a generalization of the Cartan decomposition to the non-symmetric setting. (We refer the reader to [1], [2], [14] and [8] and references therein for some aspects of the Cartan decomposition from geometric and group theoretic viewpoints.)

A generalization of the Cartan decomposition for symmetric pairs has been used in various contexts including analysis on symmetric spaces. However, there was no analogous result for non-symmetric cases before Kobayashi's paper [8]. Motivated by visible actions on complex manifolds [5], [6], he completely determined the pairs of Levi subgroups

$$
(L, H)=\left(U\left(n_{1}\right) \times \cdots \times U\left(n_{k}\right), U\left(m_{1}\right) \times \cdots \times U\left(m_{l}\right)\right)
$$

of the unitary group $G=U(n)$ such that the multiplication mapping $L \times O(n) \times H \rightarrow G$ is surjective. Furthermore, he developed a method to find a suitable subset $B$ of $O(n)$ which gives the following decomposition (a generalized Cartan decomposition, see [8]):

$$
G=L B H .
$$

2010 Mathematics Subject Classification. Primary 22E46; Secondary 32A37, 53C30.

Key words and phrases. Cartan decomposition, multiplicity-free representation, semisimple Lie group, flag variety, visible action, herringbone stitch.

This work was supported by Grant-in-Aid for JSPS Fellows (24.6877). 
On the other hand, Sasaki has been studying recently visible actions on a homogeneous space $G / H$ in the setting where both $G$ and $H$ are complex reductive Lie groups, and in his papers [17], [18], he gave a generalization of the Cartan decomposition $G=L B H$.

Back to the decomposition theory [8], we consider the following problems:

Let $G$ be a connected compact Lie group, $T$ a maximal torus, and $\sigma$ a Chevalley-Weyl involution of $G$ with respect to $T$. Here, we recall that an involutive automorphism $\mu$ of a connected compact Lie group $K$ is said to be a Chevalley-Weyl involution if there exists a maximal torus $T$ of $K$ such that $\mu(t)=t^{-1}$ for every $t \in T$. For example, $\sigma(g)=\bar{g}$ defines a Chevalley-Weyl involution of $G=U(n)$ with respect to the maximal torus consisting of diagonal matrices, and the fixed point subgroup $G^{\sigma}$ is given by $G^{\sigma}=O(n)$.

1) Classify all the pairs of Levi subgroups $L$ and $H$ with respect to $\mathfrak{t}$ such that the multiplication mapping $\psi: L \times G^{\sigma} \times H \rightarrow G$ is surjective.

2) Find a "good" representative $B \subset G^{\sigma}$ such that $G=L B H$ in the case $\psi$ is surjective. We call such a decomposition $G=L B H$ a generalized Cartan decomposition. Here we note that the roles of the subgroups $H$ and $L$ are symmetric.

We have studied the above problems for type B and type D groups in [20] and [21], respectively, and the compact Lie groups of the exceptional type will be treated in a separated paper. In this paper, we solve the problems for type $\mathrm{C}$ groups. In order to state the main results, we label the Dynkin diagram of type $\mathrm{C}_{n}$ as follows:

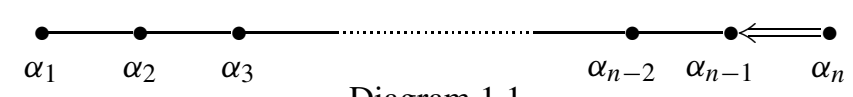

Diagram 1.1

For a subset $\Pi^{\prime}$ of the set $\Pi$ of simple roots, we denote by $\mathrm{L}_{\Pi^{\prime}}$ the Levi subgroup whose root system is generated by $\Pi^{\prime}$. For example, $\mathrm{L}_{\varnothing}$ is a maximal torus of $G$ and $\mathrm{L}_{\left\{\alpha_{p}\right\}^{c}}=$ $U(p) \times S p(n-p)$ for $G=S p(n)(1 \leq p \leq n)$. Here, we set $\operatorname{Sp}(0):=\{1\}$ for the convenience, and $\left(\Pi^{\prime}\right)^{c}$ denotes the complement $\Pi \backslash \Pi^{\prime}$.

THEOREM 1.1. Let $G$ be a connected compact simple Lie group of type $\mathrm{C}_{n}, \sigma a$ Chevalley-Weyl involution, $\Pi^{\prime}, \Pi^{\prime \prime}$ two proper subsets of $\Pi$, and $\mathrm{L}_{\Pi^{\prime}}, \mathrm{L}_{\Pi^{\prime \prime}}$ the corresponding Levi subgroups. Then the following two conditions on $\left\{\Pi^{\prime}, \Pi^{\prime \prime}\right\}$ are equivalent.

(i) $G=\mathrm{L}_{\Pi^{\prime}} G^{\sigma} \mathrm{L}_{\Pi^{\prime \prime}}$.

(ii) One of the following conditions holds up to switch of the factors $\Pi^{\prime}$ and $\Pi^{\prime \prime}$ :

Case I. $\quad\left(\Pi^{\prime}\right)^{c}=\left\{\alpha_{n}\right\}, \quad\left(\Pi^{\prime \prime}\right)^{c}=\left\{\alpha_{n}\right\}$.

Case II. $\quad\left(\Pi^{\prime}\right)^{c}=\left\{\alpha_{1}\right\}, \quad\left(\Pi^{\prime \prime}\right)^{c}=\left\{\alpha_{i}\right\}, \quad 1 \leq i \leq n$.

In the case where $G$ is simply connected, i.e., $G=S p(n)$, Theorem 1.1 means that the pairs $\left(\mathrm{L}_{\Pi^{\prime}}, \mathrm{L}_{\Pi^{\prime \prime}}\right)$ satisfying (i) are classified as follows:

Case I. $\quad\left(\mathrm{L}_{\Pi^{\prime}}, \mathrm{L}_{\Pi^{\prime \prime}}\right)=(U(n), U(n))$.

Case II . $\quad\left(\mathrm{L}_{\Pi^{\prime}}, \mathrm{L}_{\Pi^{\prime \prime}}\right)=(U(1) \times S p(n-1), U(i) \times S p(n-i)), \quad 1 \leq i \leq n$. 
In each of the two cases in Theorem 1.1, we give a generalized Cartan decomposition $G=L B H$ explicitly with $B \subset G^{\sigma}$. In Case I, $B$ is an abelian subgroup of dimension $n$, and in Case II, $B$ is given by $B=T \cdot T^{\prime} \cdot T^{\prime \prime}=\left\{x y z \in G ; x \in T, y \in T^{\prime}, z \in T^{\prime \prime}\right\}$ or $B=T^{\prime} \cdot T^{\prime \prime}=\left\{y z \in G ; y \in T^{\prime}, z \in T^{\prime \prime}\right\}$, where $T, T^{\prime}$ and $T^{\prime \prime}$ denote one-dimensional abelian subgroups. This is stated in Propositions 3.2 and 3.3. Here, we note that $B$ is no longer a subgroup in Case II.

A generalized Cartan decomposition $G=L B H$ implies that the subgroup $L$ acts on $G / H$ in a (strongly) visible fashion, and likewise $H$ on $G / L$, and $G$ on $(G \times G) /(L \times H)$. Then Kobayashi's theory leads us to three multiplicity-free theorems (triunity in [5]):

$$
\begin{array}{ll}
\text { Restriction } G \downarrow L & :\left.\operatorname{Ind}_{H}^{G}\left(\boldsymbol{C}_{\lambda}\right)\right|_{L}, \\
\text { Restriction } G \downarrow H & :\left.\operatorname{Ind}_{L}^{G}\left(\boldsymbol{C}_{\lambda}\right)\right|_{H}, \\
\text { Tensor product } & : \operatorname{Ind}_{H}^{G}\left(\boldsymbol{C}_{\lambda}\right) \otimes \operatorname{Ind}_{L}^{G}\left(\boldsymbol{C}_{\mu}\right) .
\end{array}
$$

Here $\operatorname{Ind}_{H}^{G}\left(\boldsymbol{C}_{\lambda}\right)$ denotes a holomorphically induced representation of $G$ from a character $\boldsymbol{C}_{\lambda}$ of $H$ by the Borel-Weil theorem. See [5], [6] and [7] for the general theory on the application of visible actions (including the vector bundle setting), and Corollaries 5.5 and 5.6 in this paper for type $\mathrm{C}$ groups.

Let us compare the present results with the previous results for type A [8] and type D [21]. Unlike type $\mathrm{A}$ and type $\mathrm{D}$ groups, there are only a few pairs $(L, H)$ of Levi subgroups such that $G=L G^{\sigma} H$ holds for type $\mathrm{C}$ groups. This feature in the type $\mathrm{C}$ case is similar to that in the type B case which we have discussed in [20].

This paper is organized as follows. In Section 2, we give a matrix realization of the symplectic group $G=S p(n)$ and its subgroups which are used in Sections 3 and 4 . In Section 3, we prove that (ii) implies (i). Furthermore, we find explicitly a slice $B$ that gives a generalized Cartan decomposition $G=\mathrm{L}_{\Pi^{\prime}} B \mathrm{~L}_{\Pi^{\prime \prime}}$. The converse implication (ii) $\Rightarrow$ (i) is proved in Section 4 by using the invariant theory for quivers. An application to multiplicityfree representations is discussed in Section 5.

Acknowledgment. The author would like to express his sincere gratitude to Professor Toshiyuki Kobayashi for much advice and encouragement. He would also like to show his appreciation to Dr. Atsumu Sasaki, Mr. Takayuki Okuda and Mr. Yoshiki Oshima for all the help they gave him.

2. Matrix realization. The surjectivity of $\psi: L \times G^{\sigma} \times H \rightarrow G$ depends on neither the coverings of the group $G$ nor the choice of Cartan subalgebras and Chevalley-Weyl involutions. Thus, we may and do work with the symplectic group $S p(n)$, and a fixed pair of a Cartan subalgebra and a Chevalley-Weyl involution as below.

Throughout this paper, we realize $G=S p(n)$ as the matrix group

$$
G:=\left\{g \in S L(2 n, C) ;{ }^{t} g J_{n} g=J_{n},{ }^{t} \bar{g} g=I_{2 n}\right\},
$$


where ${ }^{t} g$ denotes the transpose of $g$, and $J_{n}$ is defined by

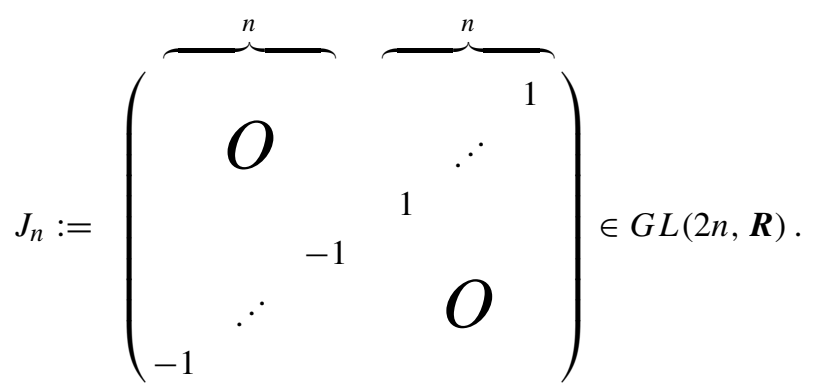

Then, the corresponding Lie algebra $\mathfrak{g}$ of $\mathrm{G}$ is given by

$$
\mathfrak{g}:=\left\{X \in \mathfrak{s l}(2 n, C) ;{ }^{t} X J_{n}+J_{n} X=O,{ }^{t} \bar{X}+X=O\right\} .
$$

We take a Cartan subalgebra $\mathfrak{t}$ of $\mathfrak{g}$ and a involution $\sigma$ as

$$
\begin{aligned}
& \mathfrak{t}=\bigoplus_{1 \leq i \leq n} \boldsymbol{R} \sqrt{-1} H_{i}, \\
& \sigma: G \rightarrow G, \quad g \mapsto \bar{g},
\end{aligned}
$$

where $H_{i}:=\mathrm{E}_{i, i}-\mathrm{E}_{2 n+1-i, 2 n+1-i}$, and $\bar{g}$ denotes the complex conjugation of $g \in G$. The differential of $\sigma$ is denoted by the same letter. Then, $\sigma$ is a Chevalley-Weyl involution of $G$ with respect to $\mathfrak{t}$. We let $\left\{\varepsilon_{i}\right\}_{1 \leq i \leq n} \subset\left(\mathfrak{t} \otimes_{\boldsymbol{R}} \boldsymbol{C}\right)^{*}$ be the dual basis of $\left\{H_{i}\right\}_{1 \leq i \leq n}$. Then we define a set of simple roots $\Pi:=\left\{\alpha_{1}, \ldots, \alpha_{n}\right\}$ by

$$
\alpha_{1}:=\varepsilon_{1}-\varepsilon_{2}, \ldots, \alpha_{n-1}:=\varepsilon_{n-1}-\varepsilon_{n}, \quad \alpha_{n}:=2 \varepsilon_{n} .
$$

Let $n=n_{1}+\cdots+n_{k}$ be a partition of $n$ with $n_{1}, \ldots, n_{k-1}>0$ and $n_{k} \geq 0$. We put

$$
\begin{aligned}
s_{i} & :=\sum_{1 \leq p \leq i} n_{p} \quad(1 \leq i \leq k-1), \\
\Pi^{\prime} & :=\Pi \backslash\left\{\alpha_{s_{i}} \in \Pi ; 1 \leq i \leq k-1\right\},
\end{aligned}
$$

and denote by $\mathrm{L}_{\Pi^{\prime}}$ the Levi subgroup whose root system is generated by $\Pi^{\prime}$. In the matrix realization, $\mathrm{L}_{\Pi^{\prime}}$ takes the form:

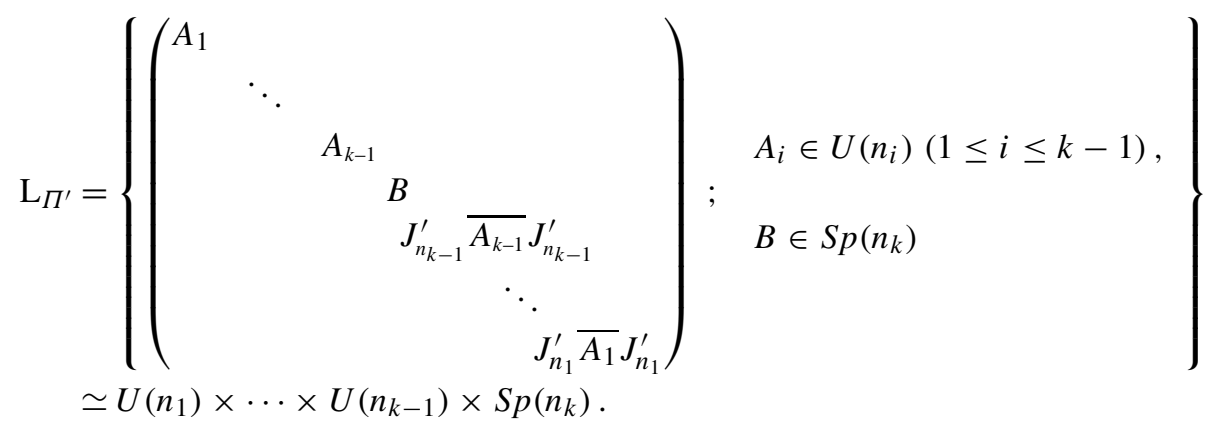


Here, all entries in the blank space are zero, and $J_{m}^{\prime}$ is defined by

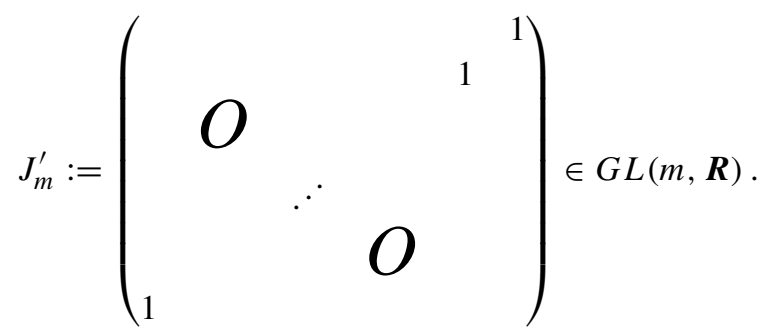

We note that the pair $\left(G, \mathrm{~L}_{\Pi^{\prime}}\right)$ forms a symmetric pair if and only if $\left(\Pi^{\prime}\right)^{c}=\left\{\alpha_{n}\right\}$. For a later purpose, we give an explicit involution $\mu$ of $G$ of which the fixed point subgroup is $\mathrm{L}_{\left\{\alpha_{n}\right\}^{c}}$. We set

$$
\mathrm{L}_{\left\{\alpha_{n}\right\}^{c}}=G^{\mu}, \quad \mu: G \rightarrow G, \quad g \mapsto I_{n, n} g I_{n, n},
$$

where $I_{n, n}$ is defined by $I_{n, n}:=\operatorname{diag}(\underbrace{1, \ldots, 1}_{n}, \underbrace{-1, \ldots,-1}_{n})$.

To obtain a generalized Cartan decomposition by the herringbone stitch method [8], we will use the following symmetric subgroup $(1 \leq i \leq n)$.

$$
\begin{aligned}
& S p(i) \times S p(n-i)= \\
&\left\{\left(\begin{array}{l|l|l}
A & & B \\
\hline & S & \\
\hline C & & D
\end{array}\right) ;\left(\begin{array}{ll}
A & B \\
C & D
\end{array}\right) \in \operatorname{Sp}(i), S \in \operatorname{Sp}(n-i)\right\} .
\end{aligned}
$$

3. Generalized Cartan decomposition. The aim of this section is to prove the implication (ii) $\Rightarrow$ (i) in Theorem 1.1. The main tool for our proof is the herringbone stitch method that reduces unknown decompositions for non-symmetric pairs to the known Cartan decomposition for symmetric pairs.

3.1. Symmetric case (Decomposition for Case I). In this subsection we recall a well-known fact on the Cartan decomposition for the symmetric case [2, Theorem 6.10], [15, Theorem 1], and give a generalized Cartan decomposition for Case I.

FACT 3.1. Let $K$ be a connected compact Lie group with Lie algebra $\mathfrak{k}$ and two involutions $\tau, \tau^{\prime}\left(\tau^{2}=\left(\tau^{\prime}\right)^{2}=\mathrm{id}\right)$. Let $H$ and L be subgroups of $K$ such that

$$
\left(K^{\tau}\right)_{0} \subset L \subset K^{\tau} \quad \text { and } \quad\left(K^{\tau^{\prime}}\right)_{0} \subset H \subset K^{\tau^{\prime}} .
$$

Here $F_{0}$ denotes the connected component of $F$ containing the identity element for a Lie group $F$. We take a maximal abelian subspace $\mathfrak{b}$ in

$$
\mathfrak{k}^{-\tau,-\tau^{\prime}}:=\left\{X \in \mathfrak{k} ; \tau(X)=\tau^{\prime}(X)=-X\right\}
$$

and write $B$ for the connected abelian subgroup with Lie algebra $\mathfrak{b}$.

Suppose that $\tau \tau^{\prime}$ is semisimple on the center $\mathfrak{z}$ of $\mathfrak{k}$. Then we have

$$
K=L B H \text {. }
$$


We shall apply Fact 3.1 to Case I. Let us set

$$
G=\operatorname{Sp}(n), \quad\left(\Pi^{\prime}\right)^{c}=\left\{\alpha_{n}\right\} .
$$

(See Diagram 1.1 for the label of the Dynkin diagram.) Then, $\left(G, \mathrm{~L}_{\Pi^{\prime}}\right)$ is a symmetric pair with $\mu$ the corresponding involution (see (2.5) for the definition of $\mu$ ). We take a maximal abelian subspace $\mathfrak{b}$ of $\mathfrak{g}^{-\mu}$ as

$$
\mathfrak{b}:=\bigoplus_{1 \leq i \leq n} \boldsymbol{R}\left(\mathrm{E}_{i, 2 n+1-i}-\mathrm{E}_{2 n+1-i, i}\right) .
$$

We note that $\mathfrak{b}$ is fixed by the Chevalley-Weyl involution $\sigma$. Using Fact 3.1, we obtain the following proposition.

Proposition 3.2 (Generalized Cartan decomposition for Case I). Let $G, \mathrm{~L}_{\Pi^{\prime}}$ be as in (3.1) and $B:=\exp (\mathfrak{b})$ for $\mathfrak{b}$ as in (3.2). Then we have

$$
G=\mathrm{L}_{\Pi^{\prime}} B \mathrm{~L}_{\Pi^{\prime}} \text {. }
$$

3.2. Decomposition for Case II. This subsection is devoted to showing the following proposition.

Proposition 3.3 (Generalized Cartan decomposition for Case II). Let $G$ be the symplectic group $\operatorname{Sp}(n)$, and $\left(\Pi^{\prime}\right)^{c}=\left\{\alpha_{1}\right\},\left(\Pi^{\prime \prime}\right)^{c}=\left\{\alpha_{i}\right\}(1 \leq i \leq n)$. We define an abelian subgroup $B^{\prime}$ and a subset $B^{\prime \prime}$ by

$$
\begin{aligned}
B^{\prime} & := \begin{cases}\exp \left(\boldsymbol{R}\left(\mathrm{E}_{1, i+1}-\mathrm{E}_{2 n-i, 2 n}-\mathrm{E}_{i+1,1}+\mathrm{E}_{2 n, 2 n-i}\right)\right) & (1 \leq i<n), \\
I_{2 n} & (i=n),\end{cases} \\
B^{\prime \prime} & :=\exp (\boldsymbol{R} X) \exp (\boldsymbol{R} Y)
\end{aligned}
$$

for $X:=\mathrm{E}_{1,2 n+1-i}+\mathrm{E}_{i, 2 n}-\mathrm{E}_{2 n+1-i, 1}-\mathrm{E}_{2 n, i}$ and $Y:=\mathrm{E}_{1,2 n}-\mathrm{E}_{2 n, 1}$. Then we have $G=\mathrm{L}_{\Pi^{\prime}} B^{\prime} B^{\prime \prime} \mathrm{L}_{\Pi^{\prime \prime}}$.

We put $L:=\mathrm{L}_{\left\{\alpha_{1}\right\}^{c}}, H:=\mathrm{L}_{\left\{\alpha_{i}\right\}^{c}}$ for simplicity. To prove Proposition 3.3, we shall show three lemmas. First, we consider the double coset decomposition of $G$ by $L$ and a symmetric subgroup $G^{\prime} G^{\prime \prime}=S p(i) \times S p(n-i)$ containing $H$, where $G^{\prime}$ and $G^{\prime \prime}$ are given by $G^{\prime}:=S p(i) \times I_{2 n-2 i}$ and $G^{\prime \prime}:=I_{2 i} \times S p(n-i)\left(\right.$ see $(2.6)$ for the realization of $\left.G^{\prime} G^{\prime \prime}\right)$.

LEMMA 3.4. The equality $G=L B^{\prime} G^{\prime} G^{\prime \prime}$ holds.

Proof. If $i=n$, both $(G, L)$ and $\left(G, G^{\prime} G^{\prime \prime}\right)$ are symmetric and thus the lemma is followed by Fact 3.1. Let us suppose $i \neq n$. We identify $G / L$ with $C P^{2 n-1}$ in the natural way (which is induced from the natural action of $G$ on $\boldsymbol{C}^{2 n}$ ). For any $x \in C P^{2 n-1}$, since $S^{4 i-1}$ and $S^{4 n-4 i-1}$ admit transitive actions of $G^{\prime}$ and $G^{\prime \prime}$, respectively, there exist $g=g^{\prime} g^{\prime \prime} \in G^{\prime} G^{\prime \prime}$ and $\theta \in \boldsymbol{R}$ such that

$$
g \cdot x=[\cos \theta: 0: \cdots: 0: \stackrel{i+1}{\sin \theta}: 0: \cdots: 0] \in B^{\prime} \cdot \overline{e_{1}},
$$

where $\overline{e_{1}}:=[1: 0: \cdots: 0] \in C P^{2 n-1}$. Thus we obtain $G=L B^{\prime} G^{\prime} G^{\prime \prime}$.

Next, we consider the double coset decomposition of $G^{\prime}$ by $\left(G^{\prime}\right)^{\mu}$ and $L \cap G^{\prime}$. 
LEMMA 3.5. The equality $G^{\prime}=\left(G^{\prime}\right)^{\mu}\left(B^{\prime \prime}\right)^{-1}\left(L \cap G^{\prime}\right)$ holds, where $\left(B^{\prime \prime}\right)^{-1}$ is defined by $\left(B^{\prime \prime}\right)^{-1}:=\left\{b^{-1} ; b \in B^{\prime \prime}\right\}$.

Proof. Let us identify $G^{\prime} /\left(L \cap G^{\prime}\right)$ with $C P^{2 i-1}$ in the natural way by taking $I_{2 n-2 i}$ away from $G^{\prime}=S p(i) \times I_{2 n-2 i}$. For any $z \in C P^{2 i-1}$, we write $z=\left[z^{\prime}: z^{\prime \prime}\right]$ where both $z^{\prime}$ and $z^{\prime \prime}$ have $i$ entries. Since $\left(G^{\prime}\right)^{\mu} \simeq U(i)$ acts on $S^{2 i-1}$ transitively, there exists $g \in\left(G^{\prime}\right)^{\mu}$ such that

$$
g \cdot z=[\left\|z^{\prime}\right\|: \overbrace{0: \cdots: 0}^{i-1}: w]
$$

for some $w$ with $i$ entries, where $\|\cdot\|$ denotes the usual Euclidean norm. We write $w=\left[w^{\prime}\right.$ : $\left.r e^{\sqrt{-1} \theta}\right]$ where $r, \theta \in \boldsymbol{R}$, and $w^{\prime}$ has $(i-1)$ entries. Then there is $g^{\prime} \in\left(\left(L \cap G^{\prime}\right)_{s s}\right)^{\mu}$ satisfying

$$
g^{\prime} \cdot(g z)=[\left\|z^{\prime}\right\|: \overbrace{0: \cdots: 0}^{i-1}:\left\|w^{\prime}\right\|: \overbrace{0: \cdots: 0}^{i-2}: r e^{\sqrt{-1} \theta}]
$$

since $\left(\left(L \cap G^{\prime}\right)_{s s}\right)^{\mu} \simeq U(i-1)$ acts on $S^{2 i-3}$ transitively. Here, $\left(L \cap G^{\prime}\right)_{s s}$ denotes the analytic subgroup of $L \cap G^{\prime}$ whose Lie algebra is the semisimple part of the Lie algebra of $L \cap G^{\prime}$. Let us set

$$
t:=\operatorname{diag}(e^{\sqrt{-1} \theta / 2}, \overbrace{0, \ldots, 0}^{i-2}, e^{-\sqrt{-1} \theta / 2}, e^{\sqrt{-1} \theta / 2}, \overbrace{0, \ldots, 0}^{i-2}, e^{-\sqrt{-1} \theta / 2}) \in\left(G^{\prime}\right)^{\mu} .
$$

We then obtain

$$
\begin{aligned}
t \cdot\left(g^{\prime} g z\right) & =\left[\left\|z^{\prime}\right\| e^{\sqrt{-1} \theta / 2}: 0: \cdots: 0:\left\|w^{\prime}\right\| e^{\sqrt{-1} \theta / 2}: 0: \cdots: 0: r e^{\sqrt{-1} \theta / 2}\right] \\
& =\left[\left\|z^{\prime}\right\|: 0: \cdots: 0:\left\|w^{\prime}\right\|: 0: \cdots: 0: r\right] \in\left(B^{\prime \prime}\right)^{-1} \cdot \overline{e_{1}},
\end{aligned}
$$

where $\overline{e_{1}}=[1: 0: \cdots: 0]$. Therefore we have $G^{\prime}=\left(G^{\prime}\right)^{\mu}\left(B^{\prime \prime}\right)^{-1}\left(L \cap G^{\prime}\right)$.

Noting that the centralizer of $B^{\prime}$ in $L \cap G^{\prime}$ is the subgroup $\left(L \cap G^{\prime}\right)_{s s} \simeq \operatorname{Sp}(i-1)$ of codimension 1 , we introduce the subgroup, which also centralizes $B^{\prime}$, by

$$
\hat{L}:=A \cdot\left(L \cap G^{\prime}\right)_{s s}\left(=\left\{a \cdot x \in G ; a \in A, x \in\left(L \cap G^{\prime}\right)_{s s}\right\}\right),
$$

where $A$ is defined by $A:=\exp \left(\boldsymbol{R} \sqrt{-1}\left(\mathrm{E}_{1,1}+\mathrm{E}_{i+1, i+1}-\mathrm{E}_{2 n-i, 2 n-i}-\mathrm{E}_{2 n, 2 n}\right)\right)$. By using Lemma 3.5, we obtain a decomposition of $G^{\prime} G^{\prime \prime}$ by $\hat{L}$ and $H$.

LEMMA 3.6. The equality $G^{\prime} G^{\prime \prime}=\hat{L} B^{\prime \prime} H$ holds.

Proof. By Lemma 3.5, we have

$$
\begin{array}{rlr}
G^{\prime} G^{\prime \prime} & =\left(\left(L \cap G^{\prime}\right) B^{\prime \prime}\left(G^{\prime}\right)^{\mu}\right) G^{\prime \prime} \\
& =\left(L \cap G^{\prime}\right) B^{\prime \prime} H \quad \text { by } H=\left(G^{\prime}\right)^{\mu} G^{\prime \prime} .
\end{array}
$$

Further, $\left(L \cap G^{\prime}\right) B^{\prime \prime} H$ coincides with $\hat{L} B^{\prime \prime} H$ :

$$
\left(L \cap G^{\prime}\right) B^{\prime \prime} H=\hat{L} B^{\prime \prime} H .
$$

Let us verify the equality (3.6). Let $A^{\prime}$ denote an abelian subgroup $\exp \left(\boldsymbol{R} \sqrt{-1}\left(\mathrm{E}_{i+1, i+1}-\right.\right.$ $\left.\mathrm{E}_{2 n-i, 2 n-i}\right)$ ) of $H$. Since $A^{\prime}$ centralizes both $B^{\prime \prime}$ and $L \cap G^{\prime}$, and since any element of $A$ can be written in terms of elements of the center of $L \cap G^{\prime}$ and $A^{\prime}$, the equality $\left(L \cap G^{\prime}\right) B^{\prime \prime} H=$ 
$\left(L \cap G^{\prime}\right) B^{\prime \prime}\left(A^{\prime} H\right)=A^{\prime}\left(L \cap G^{\prime}\right) B^{\prime \prime} H$ shows that $\left(L \cap G^{\prime}\right) B^{\prime \prime} H$ contains $\hat{L} B^{\prime \prime} H$. Conversely, since $A A^{\prime}$ contains the analytic subgroup of $L \cap G^{\prime}$, which corresponds to the center of the Lie algebra of $L \cap G^{\prime}$, the equality $\hat{L} B^{\prime \prime} H=A\left(L \cap G^{\prime}\right)_{s s} B^{\prime \prime}\left(A^{\prime} H\right)=A A^{\prime}\left(L \cap G^{\prime}\right)_{s s} B^{\prime \prime} H$ shows $\hat{L} B^{\prime \prime} H \supset\left(L \cap G^{\prime}\right) B^{\prime \prime} H$. Therefore we have the equality (3.6). By the two equalities (3.6) and (3.5), the lemma follows.

We are ready to give a proof of a generalized Cartan decomposition by using the herringbone stitch method [8].

Proof of Proposition 3.3. By using Lemmas 3.4 and 3.6, we have

$$
\begin{aligned}
& G=L B^{\prime} G^{\prime} G^{\prime \prime} \quad \text { by Lemma } 3.4 \\
& =L B^{\prime}\left(\hat{L} B^{\prime \prime} H\right) \quad \text { by Lemma } 3.6 \\
& =L B^{\prime} B^{\prime \prime} H \text {. }
\end{aligned}
$$

This completes the proof of the proposition.

Here is a herringbone stitch which we have used for $L \backslash G / H$ in Case II.

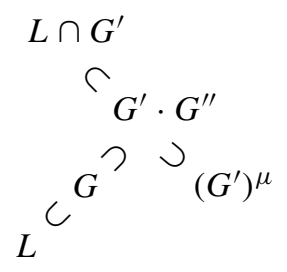

Now we have finished the proof of the implication (ii) $\Rightarrow$ (i) in Theorem 1.1 since the abelian group $B$ in Proposition 3.2 and subsets $B^{\prime}, B^{\prime \prime}$ in Proposition 3.3 are contained in $G^{\sigma}$.

4. Application of invariant theory for quivers. The aim of this section is to prove that (i) implies (ii) in Theorem 1.1. For the proof, we use invariants of quivers as in [8]. This section could be read independently of Section 3 which gives a proof on the opposite implication (ii) $\Rightarrow$ (i).

4.1. Invariants of quivers. In the following, Lemmas 4.1, 4.2 and 4.3 are parallel to [8, Lemmas 6.1, 6.2 and 6.3] respectively, and their proofs are essentially the same as that in [8]. So, we give necessary changes and precise statements, but omit the proof.

Let $\sigma: \mathrm{M}(N, \boldsymbol{C}) \rightarrow \mathrm{M}(N, \boldsymbol{C})$ be the complex conjugation with respect to $\mathrm{M}(N, \boldsymbol{R})$.

Lemma 4.1 (cf. [8, Lemma 6.1]). Let $G \subset G L(N, C)$ be a $\sigma$-stable subgroup, $R \in$ $\mathrm{M}(N, \boldsymbol{R})$ and $L$ a subgroup of $G$. If there exists $g \in G$ such that

$$
\operatorname{Ad}(L)(\operatorname{Ad}(g) R) \cap \mathrm{M}(N, \boldsymbol{R})=\emptyset,
$$

then $G \neq L G^{\sigma} G_{R}$. Here $G_{R}:=\left\{h \in G ; h R h^{-1}=R\right\}$. 
We return to the case $G=\operatorname{Sp}(n)$. We fix a partition $n=n_{1}+\cdots+n_{k}$ with $n_{i}>0$ $(1 \leq i \leq k-1), n_{k} \geq 0$ and a positive integer $r \geq 2$. We consider the following loop:

$$
i_{0} \rightarrow i_{1} \rightarrow \cdots \rightarrow i_{r}, \quad i_{s} \in\{1, \ldots, 2 k-1\}, \quad i_{0}=i_{r}, i_{s-1} \neq i_{s}(1 \leq s \leq r) .
$$

Correspondingly to this loop, we define a non-linear mapping

$$
A_{i_{0} \cdots i_{r}}: \mathrm{M}(2 n, \boldsymbol{C}) \rightarrow \begin{cases}\mathrm{M}\left(n_{i_{0}}, \boldsymbol{C}\right) & \left(i_{0}=i_{r} \neq k\right), \\ \mathrm{M}\left(2 n_{i_{0}}, \boldsymbol{C}\right) & \left(i_{0}=i_{r}=k\right)\end{cases}
$$

as follows: Let $P \in \mathrm{M}(2 n, \boldsymbol{C})$, and we write $P$ as $\left(P_{i j}\right)_{1 \leq i, j \leq 2 k-1}$ in the block matrix form corresponding to the partition $2 n=n_{1}+\cdots+n_{k-1}+2 n_{k}+n_{k-1}+\cdots+n_{1}$ of $2 n$ such that

$$
P_{i j} \in \begin{cases}\mathrm{M}\left(n_{i}, n_{j} ; \boldsymbol{C}\right) & (i, j \neq k), \\ \mathrm{M}\left(2 n_{k}, n_{j} ; \boldsymbol{C}\right) & (i=k, j \neq k), \\ \mathrm{M}\left(n_{i}, 2 n_{k} ; \boldsymbol{C}\right) & (i \neq k, j=k), \\ \mathrm{M}\left(2 n_{k}, C\right) & (i=j=k),\end{cases}
$$

where $n_{2 k-i}:=n_{i}(1 \leq i \leq k)$. Then we define $\left(\tilde{P}_{i j}\right)_{1 \leq i, j \leq 2 k-1}$ and $A_{i_{0} \cdots i_{r}}(P)$ by

$$
\tilde{P}_{i j}:= \begin{cases}P_{i j} & (i+j \leq 2 k), \\ J_{n_{i}}^{\prime t} P_{2 k-j, 2 k-i} J_{n_{j}}^{\prime} & (i+j>2 k, i, j \neq k), \\ J_{n_{k}}{ }^{t} P_{2 k-j, k} J_{n_{j}}^{\prime} & (i=k, j>k), \\ J_{n_{i}}^{\prime t} P_{k, 2 k-i} J_{n_{k}} & (i>k, j=k),\end{cases}
$$

and

$$
A_{i_{0} \cdots i_{r}}(P):=\tilde{P}_{i_{0} i_{1}} \tilde{P}_{i_{1} i_{2}} \cdots \tilde{P}_{i_{r-1} i_{r}} .
$$

For any $\ell \in L:=U\left(n_{1}\right) \times \cdots \times U\left(n_{k-1}\right) \times S p\left(n_{k}\right)$ (see (2.4) in Section 2 for the realization as a matrix), a direct computation shows

$$
\widetilde{(\operatorname{Ad}(\ell) P})_{i j}=\ell_{i} \tilde{P}_{i j} \ell_{j}^{-1} \quad(1 \leq i, j \leq 2 k-1),
$$

where $\ell_{s}(1 \leq s \leq 2 k-1)$ denotes the $(s, s)$-th block entry of $\ell$. The equality (4.3) leads us to the following lemma (cf. [8, Lemma 6.2]).

LEMMA 4.2. If there exists a loop $i_{0} \rightarrow i_{1} \rightarrow \cdots \rightarrow i_{r}$ such that at least one of the coefficients of the characteristic polynomial $\operatorname{det}\left(\lambda I_{n_{i_{0}}}-A_{i_{0} \cdots i_{r}}(P)\right)$ is not real, then

$$
\operatorname{Ad}(L) P \cap \mathrm{M}(2 n, \boldsymbol{R})=\emptyset .
$$

By Lemmas 4.1 and 4.2, we can obtain the next lemma (cf. [8, Lemma 6.3]). 
LEMMA 4.3. Let $n=n_{1}+\cdots+n_{k}$ be a partition and $L:=U\left(n_{1}\right) \times \cdots \times U\left(n_{k-1}\right) \times$ $S p\left(n_{k}\right)$ a Levi subgroup of $S p(n)$. We define a block diagonal matrix $R$ by

$$
R:=\left(\begin{array}{cccc}
R_{1} & & & \\
& R_{2} & & \\
& & \ddots & \\
& & & R_{2 k-1}
\end{array}\right),
$$

where $R_{s}, R_{2 k-s} \in \mathrm{M}\left(n_{s}, \boldsymbol{R}\right)(1 \leq s \leq k-1), R_{k} \in \mathrm{M}\left(2 n_{k}, \boldsymbol{R}\right)$ (the last condition makes sense when $n_{k} \neq 0$ ). If there exist $X \in \mathfrak{s p}(n)$ and a loop $i_{0} \rightarrow \cdots \rightarrow i_{r}$ such that

$$
\operatorname{det}\left(\lambda I_{n_{i_{0}}}-A_{i_{0} \cdots i_{r}}([X, R])\right) \notin \boldsymbol{R}[\lambda],
$$

then the multiplication map $L \times G^{\sigma} \times G_{R} \rightarrow G$ is not surjective. Here, $[X, R]:=X R-R X$.

We shall repeatedly use this lemma in the next subsection.

4.2. Necessary conditions for $G=L G^{\sigma} H$. Throughout this subsection, we set $(G, L, H)=\left(S p(n), U\left(n_{1}\right) \times \cdots \times U\left(n_{k-1}\right) \times S p\left(n_{k}\right), U\left(m_{1}\right) \times \cdots \times U\left(m_{l-1}\right) \times S p\left(m_{l}\right)\right)$, where $n=n_{1}+\cdots+n_{k}=m_{1}+\cdots+m_{l}$ with $n_{i}, m_{j}>0(1 \leq i \leq k-1,1 \leq j \leq l-1)$ and $n_{k}, m_{l} \geq 0$. We give necessary conditions on $(L, H)$ under which $G=L G^{\sigma} H$ holds. We divide the proof into three cases (Propositions 4.4 through 4.6).

Proposition 4.4. $G \neq L G^{\sigma} H$ if $k=3, l=2, m_{1}=1$.

Proposition 4.5. $G \neq L G^{\sigma} H$ if $k=l=2, n_{1}, m_{1} \geq 2, n_{2}, m_{2} \neq 0$.

Proposition 4.6. $G \neq L G^{\sigma} H$ if $k=l=2, n_{1} \geq 2, n_{2} \neq 0, m_{2}=0$.

Proof of Proposition 4.4. Let $1 \rightarrow 5 \rightarrow 2 \rightarrow 1$ be a loop. We define a diagonal matrix $R$ by $R:=\operatorname{diag}(1,0, \ldots, 0,-1) \in \mathrm{M}(2 n, \boldsymbol{R})$. Then, the centralizer $G_{R}$ coincides with $H$. We fix $u \in C$ and define $X=\left(X_{i j}\right)_{1 \leq i, j \leq 5} \in \mathfrak{s p}(n)$ in the block matrix form corresponding to the partition $2 n=n_{1}+n_{2}+2 n_{3}+n_{2}+n_{1}$ as (4.2):

$$
X_{15}:=u \mathrm{E}_{1, n_{1}} \in \mathrm{M}\left(n_{1}, \boldsymbol{C}\right), X_{41}:=\mathrm{E}_{n_{2}, 1} \in \mathrm{M}\left(n_{2}, n_{1} ; \boldsymbol{C}\right), X_{21}:=\mathrm{E}_{1,1} \in \mathrm{M}\left(n_{1}, n_{2} ; \boldsymbol{C}\right) .
$$

We define the block entries $X_{11}, X_{13}, X_{22}, X_{23}, X_{24}, X_{31}, X_{32}, X_{33}, X_{34}, X_{35}, X_{42}, X_{43}, X_{44}$, $X_{53}$ and $X_{55}$ to be zero matrices. The remaining block entries are automatically determined by the definition (2.1) of $G=S p(n)$. Then, $Q:=[X, R]$ has the following block entries:

$$
Q_{15}=-2 u \mathrm{E}_{1, n_{1}} \in \mathrm{M}\left(n_{1}, \boldsymbol{C}\right), Q_{41}=\mathrm{E}_{n_{2}, 1} \in \mathrm{M}\left(n_{2}, n_{1} ; \boldsymbol{C}\right), Q_{21}=\mathrm{E}_{1,1} \in \mathrm{M}\left(n_{1}, n_{2} ; \boldsymbol{C}\right) .
$$

By a simple matrix computation, we have (here, we recall $k=3$ )

$$
A_{1521}(Q)=Q_{15} J_{n_{1}}^{\prime t} Q_{41} J_{n_{2}}^{\prime} Q_{21}=-2 u \mathrm{E}_{1,1} \in \mathrm{M}\left(n_{1}, \boldsymbol{C}\right) .
$$

Hence we obtain

$$
\operatorname{det}\left(\lambda I_{n_{1}}-A_{1521}(Q)\right)=\lambda^{n_{1}}+2 u \lambda^{n_{1}-1} \notin \boldsymbol{R}[\lambda] \text { if } u \notin \boldsymbol{R} .
$$

By Lemma 4.3, we have shown $G \neq L G^{\sigma} H$. 
Proof OF Proposition 4.5. We may and do assume $m_{1} \geq n_{1}$ without loss of generality since the roles of $L$ and $H$ are symmetric. Let $1 \rightarrow 2 \rightarrow 3 \rightarrow 1$ be a loop, and $R=\operatorname{diag}\left(r_{1}, \ldots, r_{2 n}\right) \in \mathrm{M}(2 n, \boldsymbol{R})$ a diagonal matrix with the following entries:

$$
R:=\operatorname{diag}(\overbrace{1, \ldots, 1}^{m_{1}}, \overbrace{2, \ldots, 2}^{2 m_{2}}, \overbrace{-1, \ldots,-1}^{m_{1}}) .
$$

Then, we have $G_{R}=H$. We fix $u \in C$ and define $X=\left(X_{i j}\right)_{1 \leq i, j \leq 3} \in \mathfrak{s p}(n)$ in the block matrix form corresponding to the partition $2 n=n_{1}+2 n_{2}+n_{1}$ as (4.2):

$$
X_{12}:=\mathrm{E}_{1, n_{2}}+\mathrm{E}_{n_{1}, n_{2}+1} \in \mathrm{M}\left(n_{1}, 2 n_{2} ; \boldsymbol{C}\right), \quad X_{31}:=-\mathrm{E}_{1, n_{1}}+u \mathrm{E}_{n_{1}, 1} \in \mathrm{M}\left(n_{1}, \boldsymbol{C}\right) .
$$

We define the block entries $X_{11}, X_{22}$ and $X_{33}$ to be zero matrices. The remaining block entries of $X$ are determined automatically by (2.1). Then the block entries of $Q:=[X, R]$ are given by

$$
Q_{12}=\mathrm{E}_{1, n_{2}}+\mathrm{E}_{n_{1}, n_{2}+1} \in \mathrm{M}\left(n_{1}, 2 n_{2} ; \boldsymbol{C}\right), \quad Q_{31}=-2 \mathrm{E}_{1, n_{1}}+2 u \mathrm{E}_{n_{1}, 1} \in \mathrm{M}\left(n_{1}, \boldsymbol{C}\right) .
$$

A simple matrix computation shows (here, we recall $k=2$ )

$$
A_{1231}(Q)=Q_{12} J_{n_{2}}{ }^{t} Q_{12} J_{n_{1}}^{\prime} Q_{31}=-2 \mathrm{E}_{1, n_{1}}-2 u \mathrm{E}_{n_{1}, 1} \in \mathrm{M}\left(n_{1}, C\right),
$$

and thus we have

$$
\operatorname{det}\left(\lambda I_{n_{1}}-A_{1231}(Q)\right)=\lambda^{n_{1}}-4 u \lambda^{n_{1}-2} \notin \boldsymbol{R}[\lambda] \quad \text { if } u \notin \boldsymbol{R} .
$$

By using Lemma 4.3, we obtain $G \neq L G^{\sigma} H$.

Proof of Proposition 4.6. Let $1 \rightarrow 2 \rightarrow 3 \rightarrow 1$ be a loop, and $R$ a diagonal matrix

$$
R:=\operatorname{diag}(\overbrace{1, \ldots, 1}^{n_{1}-1},-1,-1, \overbrace{1, \ldots, 1}^{n_{2}-1}, \overbrace{-1, \ldots,-1}^{n_{2}-1}, 1,1, \overbrace{-1, \ldots,-1}^{n_{1}-1}) .
$$

Then, $G_{R}$ is conjugate to $H$ by an element of $G^{\sigma}$. We fix $u \in C$ and define $X=$ $\left(X_{i j}\right)_{1 \leq i, j \leq 3} \in \mathfrak{s p}(n)$ in the block matrix form corresponding to the partition $2 n=n_{1}+$ $2 n_{2}+n_{1}$ as (4.2):

$$
X_{12}:=u \mathrm{E}_{1,1}+\mathrm{E}_{n_{1}, 2 n_{2}} \in \mathrm{M}\left(n_{1}, 2 n_{2} ; \boldsymbol{C}\right), \quad X_{31}:=-\mathrm{E}_{1, n_{1}}-\mathrm{E}_{n_{1}, 1} \in \mathrm{M}\left(n_{1}, \boldsymbol{C}\right) .
$$

We define the block entries $X_{11}, X_{22}$ and $X_{33}$ to be zero matrices. The remaining block entries of $X$ are determined automatically by (2.1). Then $Q:=[X, R]$ has the block entries

$$
Q_{12}=-2 u \mathrm{E}_{1,1}+2 \mathrm{E}_{n_{1}, 2 n_{2}} \in \mathrm{M}\left(n_{1}, 2 n_{2} ; \boldsymbol{C}\right), Q_{31}=2 \mathrm{E}_{1, n_{1}}-2 \mathrm{E}_{n_{1}, 1} \in \mathrm{M}\left(n_{1}, \boldsymbol{C}\right) .
$$

By a simple matrix computation, we have (here, we recall $k=2$ )

$$
A_{1231}(Q)=Q_{12} J_{n_{2}}{ }^{t} Q_{12} J_{n_{1}}^{\prime} Q_{31}=-8 u \mathrm{E}_{1, n_{1}}-8 u \mathrm{E}_{n_{1}, 1} \in \mathrm{M}\left(n_{1}, C\right) .
$$

Consequently we obtain

$$
\operatorname{det}\left(\lambda I_{n_{1}}-A_{1231}(Q)\right)=\lambda^{n_{1}}-64 u^{2} \lambda^{n_{1}-2} \notin \boldsymbol{R}[\lambda] \text { if } u^{2} \notin \boldsymbol{R} .
$$

From Lemma 4.3, we have $G \neq L G^{\sigma} H$. 
4.3. Completion of the proof of Theorem 1.1. We complete the proof of the implication (i) $\Rightarrow$ (ii) in Theorem 1.1 (Proposition 4.7) by using Propositions 4.4 through 4.6. We recall that for a given partition $n=n_{1}+\cdots+n_{k}$ with $n_{1}, \ldots, n_{k-1}>0$ and $n_{k} \geq 0$, we have the corresponding Levi subgroup $\mathrm{L}_{\Pi^{\prime}}=U\left(n_{1}\right) \times \cdots \times U\left(n_{k-1}\right) \times S p\left(n_{k}\right)$ of $S p(n)$, which is associated to the subset

$$
\Pi^{\prime}:=\Pi \backslash\left\{\alpha_{i} \in \Pi ; i=\sum_{s=1}^{j} n_{s}, 1 \leq j \leq k-1\right\}
$$

of the set of simple roots $\Pi$ (see Diagram 1.1 for the label of the Dynkin diagram).

Proposition 4.7. Let $G$ be the symplectic group $S p(n), \sigma$ a Chevalley-Weyl involution, $\Pi^{\prime}, \Pi^{\prime \prime}$ subsets of the set of simple roots $\Pi$, and $\mathrm{L}_{\Pi^{\prime}}, \mathrm{L}_{\Pi^{\prime \prime}}$ the corresponding Levi subgroups. Then we have

$$
G \neq \mathrm{L}_{\Pi^{\prime}} G^{\sigma} \mathrm{L}_{\Pi^{\prime \prime}}
$$

if one of the following conditions up to switch of $\Pi^{\prime}$ and $\Pi^{\prime \prime}$ is satisfied $(1 \leq i, j, k \leq n)$ :

(I) Either $\left(\Pi^{\prime}\right)^{c}$ or $\left(\Pi^{\prime \prime}\right)^{c}$ contains more than one element.

(II) $\left(\Pi^{\prime}\right)^{c}=\left\{\alpha_{i}\right\},\left(\Pi^{\prime \prime}\right)^{c}=\left\{\alpha_{j}\right\}$ and $i, j \notin\{1, n\}$.

(III) $\left(\Pi^{\prime}\right)^{c}=\left\{\alpha_{i}\right\},\left(\Pi^{\prime \prime}\right)^{c}=\left\{\alpha_{n}\right\}$ and $i \notin\{1, n\}$.

ProOF. Let

$$
\left(\mathrm{L}_{\Pi^{\prime}}, \mathrm{L}_{\Pi^{\prime \prime}}\right)=\left(U\left(n_{1}\right) \times \cdots \times U\left(n_{k-1}\right) \times S p\left(n_{k}\right), U\left(m_{1}\right) \times \cdots \times U\left(m_{l-1}\right) \times S p\left(m_{l}\right)\right) .
$$

First, let us show the condition (I) implies (4.4). Without loss of generality, we may and do assume that $n_{1} \geq \cdots \geq n_{k-1}$ and $m_{1} \geq \cdots \geq m_{l-1}$, and that $\left(\Pi^{\prime}\right)^{c}$ contains more than one element, that is, $k \geq 3$ since the roles of $\Pi^{\prime}$ and $\Pi^{\prime \prime}$ are symmetric.

Case (I-1): $\quad m_{1}=1$. Since $\mathrm{L}_{\Pi^{\prime}}$ and $\mathrm{L}_{\Pi^{\prime \prime}}$ are contained in $U\left(n_{1}\right) \times U\left(n_{2}\right) \times S p\left(n_{3}+\right.$ $\left.\cdots+n_{k}\right)$ and $U(1) \times S p\left(m_{2}+\cdots+m_{l}\right)$, respectively, we can see that (4.4) holds by Proposition 4.4.

Case (I-2): $\quad m_{1} \geq 2, \quad n_{k} \neq 0$. Since $\mathrm{L}_{\Pi^{\prime}}$ and $\mathrm{L}_{\Pi^{\prime \prime}}$ are contained in $U\left(n_{1}+n_{2}\right) \times$ $S p\left(n_{3}+\cdots+n_{k}\right)$ and $U\left(m_{1}\right) \times S p\left(m_{2}+\cdots+m_{l}\right)$, respectively with $n_{1}+n_{2} \geq 2$ and $m_{1} \geq 2$, we can find that (4.4) holds by using Propositions 4.6 and 4.5 .

Case (I-3): $\quad m_{1} \geq 2, n_{k}=0$. In this case $n_{1} \geq 2$ and thus (4.4) follows from Propositions 4.6 and 4.5. Here, we note that $\mathrm{L}_{\Pi^{\prime}}$ and $\mathrm{L}_{\Pi^{\prime \prime}}$ are contained in $U\left(n_{1}\right) \times \operatorname{Sp}\left(n_{2}+\cdots+n_{k}\right)$ and $U\left(m_{1}\right) \times S p\left(m_{2}+\cdots+m_{l}\right)$, respectively with $n_{2} \neq 0$.

Next, let us treat the conditions (II) and (III). Then, we can immediately find that each of the conditions (II) and (III) implies (4.4) by Propositions 4.5 and 4.6, respectively.

Therefore we have finished the proof of the proposition.

5. Application of visible actions to representation theory. As an application of Theorem 1.1, we obtain some multiplicity-free theorems by using Kobayashi's theory of visible actions. Here we recall the definition [7, Definition 4.1]. 
DEFINITION 5.1. We say a biholomorphic action of a Lie group $G$ on a complex manifold $D$ is strongly visible if the following two conditions are satisfied:

(1) There exists a real submanifold $S$ such that (we call $S$ a "slice")

$$
D^{\prime}:=G \cdot S \text { is an open subset of } D .
$$

(2) There exists an antiholomorphic diffeomorphism $\sigma$ of $D^{\prime}$ such that

$$
\begin{aligned}
\left.\sigma\right|_{S} & =\operatorname{id}_{S}, \\
\sigma(G \cdot x) & =G \cdot x \quad \text { for any } x \in D^{\prime} .
\end{aligned}
$$

Definition 5.2. In the above setting, we say the action of $G$ on $D$ is $S$-visible. This terminology will be used also if $S$ is just a subset of $D$.

Let $G$ be a compact Lie group and $L, H$ its Levi subgroups. Then $G / L, G / H$ and $(G \times G) /(L \times H)$ are complex manifolds. If the triple $(G, L, H)$ satisfies $G=L G^{\sigma} H$, the following three group-actions are all strongly visible:

$$
\begin{aligned}
L & \curvearrowright G / H, \\
H & \curvearrowright G / L, \\
\Delta(G) & \curvearrowright(G \times G) /(L \times H) .
\end{aligned}
$$

Here, $\Delta(G)$ is defined by $\Delta(G):=\{(x, y) \in G \times G ; x=y\}$. The following fact [7, Theorem 4.3] constructs a family of multiplicity-free representations from visible actions.

FACT 5.3. Let $G$ be a Lie group and $\mathcal{V}$ a $G$-equivariant Hermitian holomorphic vector bundle on a connected complex manifold D. If the following three conditions from (1) to (3) are satisfied, then any unitary representation that can be embedded in the vector space $\mathcal{O}(D, \mathcal{V})$ of holomorphic sections of $\mathcal{V}$ decomposes multiplicity-freely.

(1) The action of $G$ on $D$ is $S$-visible. That is, there exist a subset $S \subset D$ and an antiholomorphic diffeomorphism $\sigma$ of $D^{\prime}$ satisfying the conditions given in Definition 5.1. Further, there exists an automorphism $\hat{\sigma}$ of $G$ such that $\sigma(g \cdot x)=\hat{\sigma}(g) \cdot \sigma(x)$ for any $g \in G$ and $x \in D^{\prime}$.

(2) For any $x \in S$, the fiber $\mathcal{V}_{x}$ at $x$ decomposes as the multiplicity free sum of irreducible unitary representations of the isotropy subgroup $G_{x}$. Let $\mathcal{V}_{x}=\bigoplus_{1 \leq i \leq n(x)} \mathcal{V}_{x}^{(i)}$ denote the irreducible decomposition of $\mathcal{V}_{x}$.

(3) $\sigma$ lifts to an antiholomorphic automorphism $\tilde{\sigma}$ of $\mathcal{V}$ and satisfies $\tilde{\sigma}\left(\mathcal{V}_{x}^{(i)}\right)=\mathcal{V}_{x}^{(i)}(1 \leq$ $i \leq n(x))$ for each $x \in S$.

We return to the case where $G=S p(n)$. The fundamental weights $\omega_{1}, \ldots, \omega_{n}$ with respect to the simple roots $\alpha_{1}, \ldots, \alpha_{n}$ are given as follows (see Diagram 1.1 for the label of the Dynkin diagram).

$$
\omega_{i}=\alpha_{1}+2 \alpha_{2}+\cdots+(i-1) \alpha_{i-1}+i\left(\alpha_{i}+\alpha_{i+1}+\cdots+\alpha_{n-1}+\frac{1}{2} \alpha_{n}\right) \quad(1 \leq i \leq n) .
$$

In the sequel, we denote by $\pi_{\lambda}$ an irreducible representation of $\operatorname{Sp}(n)$ with highest weight $\lambda=\sum_{i=1}^{n} c_{i} \omega_{i}$ with $c_{1}, \ldots, c_{n} \in N$. By using the Borel-Weil theory together with Fact 5.3 
and our generalized Cartan decompositions, we can give a geometric proof of the multiplicityfreeness property of some representations.

EXAMPLE 5.4. The tensor product $\pi_{a \omega_{n}} \otimes \pi_{b \omega_{n}+c \omega_{i}}$ is multiplicity-free for any $i(1 \leq$ $i \leq n)$ and for arbitrary non-negative integers $a, b \in N$, and $c=0$ or 1 .

To see this example, we apply Fact 5.3 to $\pi_{a \omega_{n}} \otimes \pi_{b \omega_{n}+c \omega_{i}}$ by setting $\mathcal{V}:=(\operatorname{Sp}(n) \times$ $S p(n)) \times(U(n) \times U(n))\left(C_{\omega_{n}} \otimes \Lambda^{i}\right), D:=(S p(n) \times S p(n)) /(U(n) \times U(n)), S:=B \cdot o \times\{o\}$ and $G:=\Delta(S p(n))$, where $\Lambda^{i}$ is the representation of $U(n)$ on the $i$-th alternating tensor product of $\boldsymbol{C}^{n}, B$ is as in Proposition 3.2 and $o$ denotes the identity coset. In this situation, $G_{x}$ contains $M:=\left\{\operatorname{diag}\left(\varepsilon_{1}, \ldots, \varepsilon_{n}\right) \in U(n) ; \varepsilon_{j}= \pm 1(1 \leq j \leq n)\right\}$, and $\mathcal{V}_{x}$ is given by $C_{\omega_{n}} \otimes \Lambda^{i}$ for any $x \in S$. Since $\Lambda^{i}$ is multiplicity-free as a representation of the subgroup $M$ of $U(n)$, we find that $\pi_{a \omega_{n}} \otimes \pi_{b \omega_{n}+c \omega_{i}}$ decomposes multiplicity-freely as a representation of $S p(n)$ by Fact 5.3. On the other hand, it follows from Stembridge [19] that $\pi_{a \omega_{n}} \otimes \pi_{b \omega_{n}+c \omega_{i}}$ is not multiplicity-free if $c$ is greater than one.

In the following, we confine ourselves to the line bundle case and give applications of Theorem 1.1 and Fact 5.3.

COROLlary 5.5. If the pair $(L, \lambda)$ is an entry in the Table 5-1, then the restriction $\left.\pi_{\lambda}\right|_{L}$ of the irreducible representation $\pi_{\lambda}$ of $\operatorname{Sp}(n)$ with highest weight $\lambda$ to $L$ decomposes multiplicity-freely.

\begin{tabular}{c|c} 
Levi subgroup L & highest weight $\lambda$ \\
\hline$U(n)$ & $a \omega_{n}$ \\
\hline$U(1) \times S p(n-1)$ & $a \omega_{i}$ \\
\hline$U(i) \times S p(n-i)$ & $a \omega_{1}$
\end{tabular}

TABLE 5-1.

Here, $1 \leq i \leq n$ and $a$ is an arbitrary non-negative integer.

COROLLARY 5.6. The tensor product representation $\pi_{a \omega_{1}} \otimes \pi_{b \omega_{i}}$ decomposes multiplicity freely for any non-negative integers $a, b$. Likewise, the tensor product $\pi_{a \omega_{n}} \otimes \pi_{b \omega_{n}}$ is multiplicity-free for any $a, b \in N$.

The above representations have been known to be multiplicity-free by Littelmann [13] by checking the sphericity and Stembridge [19] by a combinatorial method using character formulas. Our approach uses visible actions and is different from these two methods. We hope that further applications of Theorem 1.1 and Fact 5.3 to representation theory will be discussed in a future paper.

\section{REFERENCES}

[1] S. Helgason, Differential geometry, Lie groups and symmetric spaces, Pure Appl. Math. 80, Academic Press, New York-London, 1978.

[2] B. Hoogenboom, Intertwining functions on compact Lie groups, CWI Tract 5, Stichting Mathematisch Centrum, Centrum voor Wiskunde en Informatica, Amsterdam, 1984. 
[ 3 ] A. W. KnApp, Lie groups beyond an introduction, 2nd ed., Progr. Math. 140, Birkhäuser Boston, Inc., Boston, MA, 2002.

[4] T. Kobayashi and T. Oshima, Lie groups and representation theory (Japanese), Iwanami, 2005.

[ 5 ] T. KовAYASHI, Geometry of multiplicity-free representations of GL( $n)$, visible actions on flag varieties, and triunity, Acta Appl. Math. 81 (2004), 129-146.

[6] T. KоваYASHI, Multiplicity-free representations and visible actions on complex manifolds, Publ. Res. Inst. Math. Sci. 41 (2005), 497-549.

[ 7 ] T. KоваYASHI, Propagation of multiplicity-freeness property for holomorphic vector bundles, Progr. Math., Birkhäuser, Boston, 2013 (in press), math. RT/0607004.

[ 8 ] T. Kobayashi, A generalized Cartan decomposition for the double coset space $\left(\mathrm{U}\left(n_{1}\right) \times \mathrm{U}\left(n_{2}\right) \times \mathrm{U}\left(n_{3}\right)\right)$ $\backslash \mathrm{U}(n) /(\mathrm{U}(p) \times \mathrm{U}(q))$, J. Math. Soc. Japan 59 (2007), 669-691.

[ 9 ] T. KobAYASHI, Visible acions on symmetric spaces, Transform. Groups 12 (2007), 671-694.

[10] T. KовAYAshi, Multiplicity-free theorems of the restriction of unitary highest weight modules with respect to reductive symmetric pairs, in: Representation Theory and Automorphic Forms, 45-109, Progr. Math. 255, Birkhäuser, Boston, MA, 2008, math. RT/0607002.

[11] K. KOIKE AND I. TERADA, Young diagrammatic methods for the representation theory of the classical groups of type $B_{n}, C_{n}, D_{n}$, J. Algebra 107 (1987), 466-511.

[12] K. KoIKe AND I. TeradA, Young diagrammatic methods for the restriction of representations of complex classical Lie groups to reductive subgroups of maximal rank, Adv. Math. 79 (1990), 104-135.

[13] P. Littelmann, On spherical double cones, J. Algebra 166 (1994), 142-157.

[14] T. Matsuki, Double coset decomposition of algebraic groups arising from two involutions. I, J. Algebra 175 (1995), 865-925.

[15] T. Matsuki, Double coset decompositions of reductive Lie groups arising from two involutions, J. Algebra 197 (1997), 49-91.

[16] A. SAS AKI, Visible action on irreducible multiplicity-free spaces, Int. Math. Res. Not. IMRN (2009), 34453466.

[17] A. SASAKI, A characterization of non-tube type Hermitian symmetric spaces by visible actions, Geom. Dedicata 145 (2010), 151-158.

[18] A. SASAKI, A generalized Cartan decomposition for the double coset space $\mathrm{SU}(2 n+1) \backslash \mathrm{SL}(2 n+$ 1, C)/Sp(n, C), J. Math. Sci. Univ. Tokyo 17 (2010), 201-215.

[19] J. R. Stembridge, Multiplicity-free products and restrictions of Weyl characters, Represent. Theory 7 (2003), 404-439.

[20] Y. TANAKA, Visible actions on flag varieties of type B and a generalization of the Cartan decomposition, to appear in Bull. Aust. Math. Soc., DOI:10.1017/S0004972712000615.

[21] Y. TANAKA, Visible actions on flag varieties of type D and a generalization of the Cartan decomposition, to appear in J. Math. Soc. Japan.

[22] J. A. WOLF, Harmonic analysis on commutative spaces, Math. Surveys and Monogr. 142, American Mathematical Society, Providence, RI, 2007.

Graduate SChool of Mathematical SCIENCES

THE UNIVERSITY OF TOKYO

3-8-1, MEGURO-KU, TOKYO

$153-8914$

JAPAN

E-mail address: yuichiro@ms.u-tokyo.ac.jp 